\title{
Challenges in the Understanding and Treatment of Amyotrophic Lateral Sclerosis/Motor Neuron Disease
}

\author{
Jeffrey Rosenfeld • Michael J. Strong
}

Published online: 9 January 2015

(C) The American Society for Experimental NeuroTherapeutics, Inc. 2015

\begin{abstract}
With the acceleration in our understanding of ALS and the related motor neuron disease has come even greater challenges in reconciling all of the proposed pathogenic mechanisms and how this will translate into impactful treatments. Fundamental issues such as diagnostic definition(s) of the disease spectrum, relevant biomarkers, the impact of multiple novel genetic mutations and the significant effect of symptomatic treatments on disease progression are all areas of active investigation. In this review, we will focus on these key issues and highlight the challenges that confront both clinicians and basic science researchers.
\end{abstract}

Keywords Motor neuron disease $\cdot$ Biomarkers $\cdot$ Disease heterogeneity $\cdot$ Genetic markers $\cdot$ Clinical spectrum

\section{Introduction}

Since the earliest description in the mid 1800 s, our understanding, diagnosis, and management of patients with amyotrophic lateral sclerosis (ALS) and the related motor neuron diseases (MNDs) has progressed dramatically [1-5]. The rate of discovery of novel pathogenic mechanisms, new

Electronic supplementary material The online version of this article (doi:10.1007/s13311-014-0332-8) contains supplementary material, which is available to authorized users.

J. Rosenfeld $(\bowtie)$

Central California Neuroscience Institute, UCSF Fresno,

Division of Neurology, Fresno, CA, USA

e-mail: jrosenfeld@comcast.net

M. J. Strong

Department of Clinical Neurological Sciences, Schulich School of Medicine \& Dentistry, Western University,

London, ON N6A 5A5, Canada phenotypes, mutations, and therapeutic interventions has escalated more in the last 15 years than ever before. However, with this acceleration in our understanding of ALS/MND has come even greater challenges in reconciling all of the proposed pathogenic mechanisms and how this will translate into effective treatments that will fundamentally alter the disease course.

With our evolving understanding of the true complexity of ALS/MND has come the realization that ALS is itself not a single disease entity, but rather a heterogeneous clinical population that is best considered as a syndrome [6]. Our inability to find effective therapeutics that fundamentally alter the disease course of ALS/MND is often attributed to this heterogeneity of the patient population; thus, there is a need for better diagnostic definitions that will allow for the prognostically relevant categorizations of the disease [3-5, 7-11]. Our difficulty in identifying reliable biomarkers of disease progression and therapeutic benefit further confound this challenge [4, 12-14]. Identification of overlap in the clinical spectrum of frontotemporal dysfunction and ALS/MND has not only widened the clinical heterogeneity and highlighted new mechanisms of molecular pathology, but introduced an important prognostic element [15-18]. The discovery of novel mutations, gene products and molecular mechanisms associated with ALS/MND has challenged our concepts of how disease processes can not only be initiated, but also how such pathological processes can be propagated between individual cells in a noncell-autonomous manner [19-24].

The use of intensive symptomatic and supportive care for patients with ALS/MND is becoming more widespread and the standard of care is improving. The disease-modifying effect of such supportive care is significant and often results in improvement of both survival and function, which can exceed the treatment effect that was sought in many of our recent clinical trials [25-34]. The use of such treatment strategies introduces an 
important prognostic variable that is not consistently considered in clinical trial design and thus confounds our ability to detect efficacious therapies $[11,35]$.

In this review, we will focus on several of these key issues and highlight the challenges that confront both health professionals and researchers in this rapidly evolving area of investigation.

\section{Diagnostic Definitions}

Since the earliest descriptions of ALS/MND, the clinical triad of upper motor neuron pathology, lower motor neuron pathology, and progression has been maintained as the backbone of our diagnostic criteria [2]. There are well-established criteria for patients in whom the manifestations are restricted to dysfunction solely of the upper motor neuron (primary lateral sclerosis), lower motor neuron [progressive muscular atrophy (PMA)], or bulbar segment (progressive bulbar palsy) [8, 36-39]. There are, however, no established criteria for a significant number of patients who present and progress with predominantly upper and only subtle lower motor pathology, or, conversely, predominantly lower motor with only subtle upper motor neuron signs. These patient groups can be further prognostically dichotomized based on the presence or absence of either bulbar or respiratory dysfunction at the time of symptom onset, thus expanding the heterogeneous nature of the disease $[8,32,40]$. This variability in clinical manifestation at the time of initial assessment may be associated with significant variability in survivorship. Superimposed on this clinical variability is the presence or absence of a syndrome of frontotemporal dysfunction that may coexist with many of the motor neuron disorders and, for several, be of prognostic importance [32].

Formal diagnostic criteria for ALS/MND have been developed and modified several times [19, 41-47]. The intent of these guidelines was primarily to standardize research criteria and ensure more homogeneous patient populations for study. The use of these criteria, however, has been more effective at categorizing our degree of diagnostic certainty of a "motor neuron disease" (possible, probable, laboratory-supported, or definite) rather than identifying a homogeneous group of patients or specific disease subtypes. The result has been a wide range of phenotypic expression included under the diagnostic definition of ALS/MND. Several attempts to categorize patients identified as having "ALS/MND" have been attempted [48].

The onset of ALS/MND is often regionally defined with subsequent spread to other contiguous areas [49-51]. Different patterns of spread have been described, mainly to allow us to anticipate the regional involvement and prognosis [50, 52, 53]. Furthermore, different staging systems have been devised to account for this regional spread $[54,55]$. None of these efforts, however, have progressed to the point of identifying more homogeneous disease cohorts based upon region of onset or subsequent spread that can then be applied to the design of clinical trials.

In the absence of widespread acceptance of diagnostic criteria for specific disease subtypes, there have been a number of clinical phenotypes described [11, 37, 48, 56-59]. These phenotypic descriptions, with variable disease progression, severity, and survival often fit into the basic diagnostic criteria of ALS/MND, although they do not necessarily obey the more classically described patterns of regional spread involving upper and lower motor neuron pathology. Some of these "ALS" phenotypes have even included disorders already identified with distinct labels such as PMA or progressive bulbar atrophy (PBA) [48]. In addition, many of these patients with "ALS" may maintain a regionally restricted involvement for prolonged periods.

As alluded to above, the presence or absence of frontotemporal dysfunction is an important prognostic variable in patients with all types of ALS/MND. While frontotemporal dysfunction has been observed across a spectrum of pure lower motor neuron disorders, including X-linked spinal and bulbar muscular atrophy (Kennedy syndrome) and PMA, there are no prospective studies addressing the impact of frontotemporal dysfunction on survival in these entities [60-62]. International consensus criteria have been developed that provide a framework in which patients with ALS/MND can be described as being pure ALS in the absence of any evidence of frontotemporal dysfunction, as having a frontotemporal dementia (FTD) consistent with either the Hodge or Neary criteria for FTD (ALS-FTD), or as having either a cognitive (ALSci) and/or behavioral manifestation (ALSbi) that do not fulfill the criteria for FTD [47]. When these criteria are applied in a prospective manner, approximately half of all patients will have no evidence of frontotemporal dysfunction, between $2 \%$ and $4 \%$ will have Alzheimer's disease, 25-30 \% will have ALSci, and the remaining will have a behavioral syndrome [63]. There has been considerable effort in further clarifying those patients who suffer from either ALSci, ALSbi, or an overlap between the 2, which has been often described as a dysexecutive syndrome. The importance in doing so relates specifically to the impact that these syndromes have on survival.

ALSci is most consistently associated with alterations in verbal fluency and the generation of action verbs, while ALSbi can have one of many manifestations, including apathy, behavioral disinhibition, irritability, loss of sympathy or empathy, perseverative or stereotypic behavior, or alterations in eating behavior [64]. The latter are best identified through the use of one of several available screening tools that incorporate caregiver input [65-67]. Although the presence of psychotic symptoms has been highlighted by the recent identification of C9orf72 pathological hexanucleotide expansions in both ALS and FTD, psychotic symptoms are uncommon in ALS. The dysexecutive syndrome of ALS seems to best correlate with deficits in theory of mind, reflecting the 
capacity to appreciate other's intentions and beliefs, and to extend this into predicting other's behavior by attributing an independent mental state to them. Such deficits are reflective of orbitofrontal and anterior cingulate pathology and can be observed in upwards of $50 \%$ of patients with ALS who have no evidence otherwise of dementia $[68,69]$. When impairment in social cognition is considered along with ALSci, ALSbi, and ALS-FTD, a more robust picture of the prevalence of frontotemporal dysfunction in ALS is attained in which executive dysfunction is second to only ALSci as being the most common manifestation. Those individuals with evidence of executive or behavioral dysfunction have a significantly more rapid disease course [16, 70].

\section{Biomarkers}

The multisystem involvement of patients affected by ALS/ MND, as well as the varied mechanisms attributed to the disease has led to a multitude of clinical and biological markers proposed as indices of disease progression and/or severity $[4,12,14]$. Despite this, one of the primary challenges in studying ALS/MND is the absence of an accepted marker with both the appropriate sensitivity and specificity applicable to the wide range of affected patients. The result of this void in our understanding is a lack of confidence that we can reliably measure disease progression or therapeutic efficacy in our clinical trials.

Given that ALS/MND is a disease in which motor units are progressively lost, strength measures should be expected to best characterize the extent of weakness. A quantitative assessment of strength has, in fact, been included in almost every clinical trial of ALS/MND. Multiple measures of strength have been repeatedly utilized in this patient population ranging from classic categorical measurement (manual muscle testing), comprehensive composite measures of strength (Tufts Quantitative Neuromuscular Exam [71, 72]) and focused quantitative evaluation of individual muscle groups (hand-held dynamometry [73]). Despite the logic of utilizing strength as a measure of disease activity in a motor neuron disease, the variability between patients with regard to affected regions, baseline strength, and rate of progression has offset the practical utility of strength measurements in clinical trials.

Several different electrophysiological determinations of motor unit number have been refined and proposed as surrogate markers of disease progression, with good inter-rater reliability [74-76]. These estimates, however, are time consuming and still somewhat operator dependent. In addition, disease progression within ALS/MND is commonly not uniform in all muscle groups, and sampling error may further confound this technique. Variability of individual motor units after repeated stimulation is perhaps more pronounced in patients with ALS/MND. Some newer measures such as motor unit index and multipoint incremental stimulation have addressed this issue, but these methods have not yet been accepted routinely [76-78].

Composite functional measures such as the ALS Functional Rating Scale-Revised (ALSFRS-R) have been most consistently applied in both routine care and most clinical trials involving patients with ALS/MND. The score is used to evaluate symptoms related to bulbar, limb, and respiratory function. The ALSFRS-R has repeatedly been shown to be a reliable and valid measure with a predictable linear course for which the rate of change may predict survival [79-81]. This has resulted in the widespread use of the measure, facilitated by the ease in obtaining it and a large established cohort database. The ALSFRS-R, however, is more suited to detecting changes in patients in the mid-stage of disease with appendicular involvement, and it may not be an ideal measure of global function [79, $80,82]$.

Other proposed clinical biomarkers for disease progression include electrical impedance myography, muscle ultrasound, and various imaging modalities (i.e., voxel-based morphometry, diffusion tensor imaging, T2 quantification, positron emission tomography) [83-85]. Biochemical markers of underlying neuropathology phosphorylated high molecular weight neurofilament protein $(\mathrm{pNFH})$ or immunological markers are also promising [86]. These methods have generated interesting data, particularly within certain disease presentations involving upper motor neuron dysfunction, but have not yet been accepted as markers applicable throughout the disease spectrum.

\section{The Role of Molecular Biology in Understanding ALS/MND}

The potential for genetic markers as correlates of disease subtype and progression has generated much enthusiasm and promise, although remains incompletely understood. There are currently very few established relationships between a specific genetic mutation and a specific phenotype. An escalating library of data in this regard is available through an online registry (http://alsod.iop.kcl.ac.uk) [87]. The hope is that a potential etiology, at least for a specific phenotype, will emerge and highlight a primary disease mechanism [3, 5, 88, 89].

The initial discovery, 20 years ago, that a dominant missense mutation in SOD1 can account for up to $15 \%$ of familial cases offered great promise for understanding the pathophysiology of motor neuron degeneration in those patients. Currently, there have been $>100$ distinct SOD1 mutations identified with tremendous variability in the resulting phenotype. The A4V, G41S, G93A, and R115G SOD1 mutations frequently give rise to a very aggressive disease course. Conversely, the D90A SOD1 mutation is associated with a much 
more indolent course, although the clinical manifestation as ALS remains remarkably similar to the other $S O D 1$ mutations $[90,91]$. This observation serves to highlight that a single gene mutation can give rise to heterogeneous pattern of clinical deficits, supporting the syndromic nature of ALS/MND [6].

This is further highlighted by the finding that approximately two-thirds of patients with familial ALS and $10 \%$ of patients with sporadic ALS harbor a genetic mutation [92]. Increasingly, genome-wide association studies are identifying genes not suspected to be causative in ALS, but rather that have a direct impact on the disease phenotype [93, 94]. For instance, $U N C 13 \mathrm{~A}$, for which a single nucleotide polymorphism appears to confer susceptibility to ALS, also confers a shorter survival, while the KIFAP 3 and EPHA4 variants seems to predict a longer survival, including a greater than expected association of KIPAP3 with primary lateral sclerosis [95]. Other studies implicate the short arm of chromosome 1 with age of disease onset. [96]. Perhaps nowhere is this variability in the effect of a single gene mutation more obvious that the impact of a massive GGGGCC $\left(\mathrm{G}_{4} \mathrm{C}_{2}\right)$ repeat expansion in C9ORF72 in which ALS, FTD, and ALS-FTD have all been described [97]. Such striking variability also suggests the presence of multiple disease modifiers, not the least of which may be important gene-environment interactions [98].

As our understanding of the molecular pathogenesis of ALS becomes increasing clear, it is now evident that a great deal of the genetic basis of both sporadic and familial ALS can be attributed to alterations in RNA metabolism at multiple stages of the cascade of pathological changes leading to motor neuron death $[99,100]$.

\section{Advances in Treatment Affecting Disease Course}

Current clinical practice for patients affected by ALS/MND includes a combination of proactive, adjunctive and symptomatic therapies. Outcome data are lacking relative to the number and variety of treatments used regularly in this population. While treatment approaches are likely more consistent today than they were a decade ago, implementation of these often efficacious treatments has introduced another source of variability in comparing groups of patients for clinical trials. Specifically, maintenance of respiratory health, nutritional stability, physical safety, and aids to improve daily function have an impact on quality of life and disease duration. While these benefits have been difficult to measure in controlled studies, multidisciplinary care has become the standard of treatment for this complex multisystem disease. It is no longer appropriate to refer to ALS/MND as an "untreatable" disease. The magnitude of the benefit from such treatments described below often exceeds the treatment effects that are sought in clinical trials of potential therapeutic agents. These interventions have, consequently, introduced an underappreciated source of variability in studying populations of patients with ALS/MND with novel therapeutic agents.

Inadequate nutrition and weight loss are common clinical features in ALS/MND. The etiology contributing to this includes dysphagia, impaired motor function (involving bulbar and extremity muscles), and the possibility that a hypermetabolic state exists [101-104]. The association of weight loss, impaired nutrition, and shortened survival is commonly observed and actively targeted for proactive treatment [105-110]. Multiple interventions to address these significant determinants of health and longevity have implicated a multidisciplinary approach involving 1) dieticians (include altering food consistency, high-calorie supplements, and adequate hydration); 2) occupational therapy (hand braces, altered utensils, mobile arm supports, home modifications); 3) physical therapy (range of motion, bracing, seating, maintaining strength); 4) social work (home care assistance, financial support, emotional health); and 5) respiratory therapy (adequate ventilation, secretion management). Although large, controlled outcomes studies have not been completed, the benefit of such multidisciplinary care, on both survival and quality of life, has been widely accepted [111-113].

\section{Percutaneous Endoscopic Gastrostomy}

Percutaneous gastrostomy has become the primary and most significant tertiary intervention to address the weight loss and impaired nutrition common amongst the ALS/MND patient population. Early intervention, when dysphagia is mild/moderate, may be preferred as a proactive intervention to maximally affect survival and quality of life, although data supporting the survival benefit are inconsistent $[33,114,115]$. Patient acceptance is often better when the intervention is initially used as a supplement to oral caloric intake and not the sole source of nutrition. Patient and family education on the benefits of percutaneous gastrostomy often results in an improved sense of autonomy, confidence, and quality of life [115-117]. Patients and families relate that there is a sense of not being able to affect any significant change in disease course heightened by a fear of choking and the caregiver burden. Percutaneous gastrostomy can overcome these issues, although formal outcome data on the subject are difficult to collect owing to multiple confounding issues that affect an individual patient's decision to accept gastrostomy support.

The impact of nutritional intervention on survival is recognized; however, the benefit of gastrostomy alone has not been shown [118-120]. Retrospective population studies suggest that the relationship is "tentative" and other factors such as age and site of onset along with riluzole and the use of noninvasive positive pressure ventilation, may be more significant prognostic determinants. Currently, implementation of nutritional therapies remain both an opportunity and a challenge in the 
overall treatment regimen. While it is difficult to accept that patients receiving adequate nutrition and hydration do not have improved survival (relative to malnourished, dehydrated patients), the inconsistent results from retrospective analyses highlight the difficulty from drawing conclusions from a very heterogeneous patient population [121].

Current Practice Parameter guidelines published by the American Academy of Neurology recommend percutaneous gastrostomy placement before the patient's forced vital capacity falls below $50 \%$ predicted in order to avoid risk of respiratory compromise during the procedure [115]. The timing of this intervention is, however, critical as it introduces an important source of variability when considering treatment trials where functional capacity, quality of life, and survival are relevant endpoints [107, 122, 123].

\section{Respiratory Therapies}

The use of aggressive respiratory management, including control of secretions, noninvasive ventilation, and pharmacologic intervention is both necessary and efficacious in the ALS/MND patient population. In contrast to the reported data on nutritional interventions, there is a more consistent benefit demonstrated on survival and quality of life [115, 124, 125]. The timing of this respiratory intervention may introduce another source of variability on survival, quality of life, and overall function.

Secretion management is closely linked to the delivery of improved ventilation in patients with ALS/MND. Inexsufflation and use of high frequency chest wall oscillation are increasingly common and have been shown to improve ventilation [126-129]. Physical techniques such as breath stacking and assisted cough, as well as medication management (bronchodilators, anticholinergic agents), have become increasingly common in aggressive multidisciplinary treatment settings. In addition, use of intrasalivary gland injection of botulinum toxin has been shown to result in significant improvement of sialorrhea and improve respiratory insufficiency $[130,131]$.

\section{Conclusions}

The current pace of discovery and identification of novel disease mechanisms in ALS/MND is unprecedented. Despite the great expansion in our knowledge base, there remain some fundamental challenges on issues such as disease definition, appropriate biomarkers of disease progression, interpretation of the multitude of genetic mutations associated with disease, and the impact of aggressive symptomatic treatment. Identification of frontotemporal dysfunction in a majority of patients with both familial and sporadic ALS/MND has both added greatly to the potential for a common underlying mechanism between ALS and frontotemporal dysfunction, while at the same time providing an important prognostic determinant. Advances in this area, however, have also introduced challenges in the heterogeneity of diagnostic definitions affecting this patient population. Our ability to design effective clinical trials that will ultimately lead to efficacious therapies will likely depend upon resolution of some of these most basic questions of diagnosis and disease progression. By comparison with the fairly straightforward and simple initial description of ALS by Charcot in the 1800 s, we confront today a considerable complexity whose understanding may ultimately shed light on mechanisms not only of ALS/MND, but also of other neurodegenerative disorders.

Required Author Forms Disclosure forms provided by the authors are available with the online version of this article.

\section{References}

1. Jackson, C.E. and J. Rosenfeld, Motor neuron disease, in Physical Medicine \& Rehabilitation Clinics of North America. 2001. p. 33552 , ix-x.

2. Strong, M. and J. Rosenfeld, Amyotrophic lateral sclerosis: a review of current concepts. Amyotrophic Lateral Sclerosis \& Other Motor Neuron Disorders, 2003. 4(3): p. 136-43.

3. Su, X.W., J.R. Broach, J.R. Connor, G.S. Gerhard, and Z. Simmons, Genetic heterogeneity of amyotrophic lateral sclerosis: implications for clinical practice and research. Muscle \& Nerve, 2014. 49(6): p. 786-803.

4. Turner, M.R., R. Bowser, L. Bruijn, et al., Mechanisms, models and biomarkers in amyotrophic lateral sclerosis. Amyotrophic Lateral sclerosis \& Frontotemporal Degeneration, 2013. 14 Suppl 1: p. 19-32.

5. Sabatelli, M., A. Conte, and M. Zollino, Clinical and genetic heterogeneity of amyotrophic lateral sclerosis. Clinical Genetics, 2013. 83(5): p. 408-16.

6. Strong, M., The evidence for ALS as a multisystems disorder of limited phenotypic expression. Can J Neurol Sci, 2001. 28: p. 283-298.

7. Al-Chalabi, A., A. Jones, C. Troakes, et al., The genetics and neuropathology of amyotrophic lateral sclerosis. Acta Neuropathologica, 2012. 124(3): p. 339-52.

8. Sabatelli, M., M. Zollino, M. Luigetti, et al., Uncovering amyotrophic lateral sclerosis phenotypes: clinical features and long-term follow-up of upper motor neuron-dominant ALS. Amyotrophic Lateral Sclerosis, 2011. 12(4): p. 278-82.

9. Vucic, S., Heterogeneity of pathological processes in amyotrophic lateral sclerosis? Journal of Neurology, Neurosurgery \& Psychiatry, 2011. 82(8): p. 827.

10. Ravits, J. and B.J. Traynor, Current and future directions in genomics of amyotrophic lateral sclerosis. Physical Medicine \& Rehabilitation Clinics of North America, 2008. 19(3): p. 461-77, viii.

11. Beghi, E., A. Chio, P. Couratier, et al., The epidemiology and treatment of ALS: focus on the heterogeneity of the disease and critical appraisal of therapeutic trials. Amyotrophic Lateral Sclerosis, 2011. 12(1): p. 1-10.

12. Bede, P., A.L.W. Bokde, S. Byrne, et al., Spinal cord markers in ALS: diagnostic and biomarker considerations. Amyotrophic Lateral Sclerosis, 2012. 13(5): p. 407-15.

13. Bhutani, H. and A. Anand, Biomarkers in amyotrophic lateral sclerosis: is there a neurovascular pathway? Current Neurovascular Research, 2012. 9(4): p. 302-9. 
14. Bowser, R., M.R. Turner, and J. Shefner, Biomarkers in amyotrophic lateral sclerosis: opportunities and limitations. Nature Reviews Neurology, 2011. 7(11): p. 631-8.

15. Elamin, M., P. Bede, S. Byrne, et al., Cognitive changes predict functional decline in ALS: a populationbased longitudinal study. Neurology, 2013. 80(17): p. 1590-7.

16. Elamin, M., J. Phukan, P. Bede, et al., Executive dysfunction is a negative prognostic indicator in patients with ALS without dementia. Neurology, 2011. 76(14): p. 1263-9.

17. Hu, W.T., M. Shelnutt, A. Wilson, et al., Behavior matters-cognitive predictors of survival in amyotrophic lateral sclerosis. PLoS ONE [Electronic Resource], 2013. 8(2): p. e57584.

18. Olney, R.K., J. Murphy, D. Forshew, et al., The effects of executive and behavioral dysfunction on the course of ALS. Neurology, 2005. 65(11): p. 1774-7.

19. Strong, M.J., The syndromes of frontotemporal dysfunction in amyotrophic lateral sclerosis. Amyotrophic Lateral Sclerosis, 2008. 9(6): p. 323 - 338.

20. Strong, M.J., C. Lomen-Hoerth, R.J. Caselli, E.H. Bigio, and W. Yang, Cognitive impairment, frontotemporal dementia, and the motor neuron diseases. Annals of Neurology, 2003. 54(5).

21. Talbot, P.R., P.J. Goulding, J.J. Lloyd, et al., Inter-relation between "classic" motor neuron disease and frontotemporal dementia: neuropsychological and single photon emission computed tomography study. Journal of Neurology, Neurosurgery \& Psychiatry, 1995. 58(5): p. 541-7.

22. Byrne, S., M. Elamin, P. Bede, et al., Cognitive and clinical characteristics of patients with amyotrophic lateral sclerosis carrying a C9orf72 repeat expansion: a population-based cohort study.[Erratum appears in Lancet Neurol. 2012 May;11(5):388]. Lancet Neurology, 2012. 11(3): p. 232-40.

23. DeJesus-Hernandez, M., I.R. Mackenzie, B.F. Boeve, et al., Expanded GGGGCC hexanucleotide repeat in noncoding region of C9ORF72 causes chromosome 9p-linked FTD and ALS. Neuron, 2011. 72(2): p. 245-56.

24. Murray, M.E., M. DeJesus-Hernandez, N.J. Rutherford, et al., Clinical and neuropathologic heterogeneity of c9FTD/ALS associated with hexanucleotide repeat expansion in C9ORF72. Acta Neuropathologica, 2011. 122(6): p. 673-90.

25. Ashworth, N.L., L.E. Satkunam, and D. Deforge, Treatment for spasticity in amyotrophic lateral sclerosis/motor neuron disease. Cochrane Database of Systematic Reviews, 2012. 2: p. CD004156.

26. Baldinger, R., H.D. Katzberg, and M. Weber, Treatment for cramps in amyotrophic lateral sclerosis/motor neuron disease. Cochrane Database of Systematic Reviews, 2012. 4: p. CD004157.

27. Young, C.A., C. Ellis, J. Johnson, S. Sathasivam, and N. Pih, Treatment for sialorrhea (excessive saliva) in people with motor neuron disease/amyotrophic lateral sclerosis. Cochrane Database of Systematic Reviews, 2011(5): p. CD006981.

28. Carratu, P., L. Spicuzza, A. Cassano, et al., Early treatment with noninvasive positive pressure ventilation prolongs survival in Amyotrophic Lateral Sclerosis patients with nocturnal respiratory insufficiency. Orphanet Journal Of Rare Diseases, 2009. 4: p. 10.

29. Marquardt, G. and V. Seifert, Use of intrathecal baclofen for treatment of spasticity in amyotrophic lateral sclerosis. Journal of Neurology, Neurosurgery \& Psychiatry, 2002. 72(2): p. 275-6.

30. Hardiman, O., Symptomatic treatment of respiratory and nutritional failure in amyotrophic lateral sclerosis. Journal of Neurology., 2000. 247(4): p. 245-51.

31. Radunovic, A., D. Annane, M.K. Rafiq, and N. Mustfa, Mechanical ventilation for amyotrophic lateral sclerosis/motor neuron disease. Cochrane Database of Systematic Reviews, 2013. 3: p. CD004427.

32. Georgoulopoulou, E., N. Fini, M. Vinceti, et al., The impact of clinical factors, riluzole and therapeutic interventions on ALS survival: a population based study in Modena, Italy. Amyotrophic
Lateral sclerosis \& Frontotemporal Degeneration, 2013. 14(5-6): p. 338-45.

33. Katzberg, H.D. and M. Benatar, Enteral tube feeding for amyotrophic lateral sclerosis/motor neuron disease. Cochrane Database of Systematic Reviews, 2011(1): p. CD004030.

34. Wood-Allum, C. and P.J. Shaw, Motor neurone disease: a practical update on diagnosis and management. Clinical Medicine, 2010. 10(3): p. 252-8.

35. Beghi, E., T. Mennini, C. Bendotti, et al., The heterogeneity of amyotrophic lateral sclerosis: a possible explanation of treatment failure. Current Medicinal Chemistry, 2007. 14(30): p. 3185-200.

36. Rosenfeld, J. and M. Swash, What's in a name? Lumping or splitting ALS, PLS, PMA and the other motor neuron diseases. Neurology, 2006. 66(draft).

37. Ravits, J.M. and A.R. La Spada, ALS motor phenotype heterogeneity, focality, and spread: deconstructing motor neuron degeneration. Neurology, 2009. 73(10): p. 805-11.

38. Turner, M.R., J. Scaber, J.A. Goodfellow, et al., The diagnostic pathway and prognosis in bulbar-onset amyotrophic lateral sclerosis. Journal of the Neurological Sciences, 2010. 294(1-2): p. 81-5.

39. Pringle, C.E., A.J. Hudson, D.G. Munoz, et al., Primary lateral sclerosis. Clinical features, neuropathology and diagnostic criteria. Brain, 1992. 115(Pt 2): p. 495-520.

40. Tartaglia, M.C., A. Rowe, K. Findlater, et al., Differentiation between primary lateral sclerosis and amyotrophic lateral sclerosis: examination of symptoms and signs at disease onset and during follow-up. Archives of Neurology, 2007. 64(2): p. 232-6.

41. Brooks, B.R., R. Mikker, M. Swash, and T. Munsat, El Escorial revisited: Revised criteria for the diagnosis of amyotrophic lateral sclerosis. ALS and Other Motor Neuron Diseases, 2000. 1: p. 293 99.

42. Traynor, B.J., M.B. Codd, B. Corr, et al., Clinical features of amyotrophic lateral sclerosis according to the El Escorial and Airlie House diagnostic criteria: A population-based study. Archives of Neurology, 2000. 57(8): p. 1171-6.

43. Boekestein, W.A., B.U. Kleine, G. Hageman, H.J. Schelhaas, and M.J. Zwarts, Sensitivity and specificity of the 'Awaji' electrodiagnostic criteria for amyotrophic lateral sclerosis: retrospective comparison of the Awaji and revised El Escorial criteria for ALS. Amyotrophic Lateral Sclerosis, 2010. 11(6): p. 497-501.

44. Carvalho, M.D. and M. Swash, Awaji diagnostic algorithm increases sensitivity of El Escorial criteria for ALS diagnosis. Amyotrophic Lateral Sclerosis, 2009. 10(1): p. 53-7.

45. Costa, J., M. Swash, and M. de Carvalho, Awaji criteria for the diagnosis of amyotrophic lateral sclerosis:a systematic review. Archives of Neurology, 2012. 69(11): p. 1410-6.

46. Higashihara, M., M. Sonoo, I. Imafuku, et al., Fasciculation potentials in amyotrophic lateral sclerosis and the diagnostic yield of the Awaji algorithm. Muscle \& Nerve, 2012. 45(2): p. 175-82.

47. Strong, M.J., G.M. Grace, M. Freedman, et al., Consensus criteria for the diagnosis of frontotemporal cognitive and behavioural syndromes in amyotrophic lateral sclerosis.[Erratum appears in Amyotroph Lateral Scler. 2009 Aug;10(4):252]. Amyotrophic Lateral Sclerosis, 2009. 10(3): p. 131-46.

48. Swash, M. and J. Desai, Motor neuron disease: Classification and nomenclature. Amyotrophic Lateral Sclerosis, 2000. 1(2): p. 105 112.

49. Gargiulo-Monachelli, G.M., F. Janota, M. Bettini, et al., Regional spread pattern predicts survival in patients with sporadic amyotrophic lateral sclerosis. European Journal of Neurology, 2012. 19(6): p. 834-41.

50. Ravits, J., P. Laurie, Y. Fan, and D.H. Moore, Implications of ALS focality: rostral-caudal distribution of lower motor neuron loss postmortem. Neurology, 2007. 68(19): p. 1576-82. 
51. Ravits, J., P. Paul, and C. Jorg, Focality of upper and lower motor neuron degeneration at the clinical onset of ALS. Neurology, 2007. 68(19): p. 1571-5.

52. Fujimura-Kiyono, C., F. Kimura, S. Ishida, et al., Onset and spreading patterns of lower motor neuron involvements predict survival in sporadic amyotrophic lateral sclerosis. Journal of Neurology, Neurosurgery \& Psychiatry, 2011. 82(11): p. 1244-9.

53. Sekiguchi, T., T. Kanouchi, K. Shibuya, et al., Spreading of amyotrophic lateral sclerosis lesions-multifocal hits and local propagation? Journal of Neurology, Neurosurgery \& Psychiatry, 2014. 85(1): p. 85-91.

54. Balendra R, Jones A, Jivraj N, and e. al, Use of clinical staging in amyotrophic lateral sclerosis for phase 3 clinical trials. J Neurol Neurosurg Psychiatry, 2014. 24(doi 10.1136/jmp-213-306865).

55. Roche, J.C., R. Rojas-Garcia, K.M. Scott, et al., A proposed staging system for amyotrophic lateral sclerosis. Brain, 2012. 135(Pt 3): p. 847-52.

56. Swash, M. and J. Desai, Motor neuron disease: classification and nomenclature. Amyotrophic Lateral Sclerosis \& Other Motor Neuron Disorders, 2000. 1(2): p. 105-12.

57. Chio, A., A. Calvo, C. Moglia, et al., Phenotypic heterogeneity of amyotrophic lateral sclerosis: a population based study. Journal of Neurology, Neurosurgery \& Psychiatry, 2011. 82(7): p. 740-6.

58. Hu, M., C. Ellis, A. Al-Chalabi, P. Leigh, and C. Shaw, Flail arm syndrome: a distinctive variant of amyotrophic lateral sclerosis. J Neurol Neurosurg Psychiatry, 1998. 65: p. 950-951.

59. Kobayashi, Z., K. Tsuchiya, T. Arai, et al., Pseudopolyneuritic form of ALS revisited: clinical and pathological heterogeneity. Neuropathology, 2010. 30(4): p. 372-80.

60. Lambrechts, D., W. Robberecht, and P. Carmeliet, Heterogeneity in motoneuron disease. p. 536-44, 2007 Oct.

61. Kasper, E., M. Wegrzyn, I. Marx, et al., Minor cognitive disturbances in X-linked spinal and bulbar muscular atrophy, Kennedy's disease. Amyotrophic Lateral sclerosis \& Frontotemporal Degeneration, 2014. 15(1-2): p. 15-20.

62. Raaphorst, J., M. de Visser, M.-J. van Tol, et al., Cognitive dysfunction in lower motor neuron disease: executive and memory deficits in progressive muscular atrophy. Journal of Neurology, Neurosurgery \& Psychiatry, 2011. 82(2): p. 170-5.

63. Soukup, G.R., A.-D. Sperfeld, I. Uttner, et al., Frontotemporal cognitive function in X-linked spinal and bulbar muscular atrophy (SBMA): a controlled neuropsychological study of 20 patients. Journal of Neurology, 2009. 256(11): p. 1869-75.

64. Consonni, M., S. Iannaccone, C. Cerami, et al., The cognitive and behavioural profile of amyotrophic lateral sclerosis: application of the consensus criteria. Behavioural Neurology, 2013. 27(2): p. 14353.

65. Raaphorst, J., M. de Visser, W.H.J.P. Linssen, R.J. de Haan, and B. Schmand, The cognitive profile of amyotrophic lateral sclerosis: A meta-analysis. Amyotrophic Lateral Sclerosis, 2010. 11(1-2): p. 2737.

66. Grossman, A.B., S. Woolley-Levine, W.G. Bradley, and R.G. Miller, Detecting neurobehavioral changes in amyotrophic lateral sclerosis. Amyotrophic Lateral Sclerosise, 2007. 8(1): p. 56-61.

67. Lillo, P., E. Mioshi, M.C. Zoing, M.C. Kiernan, and J.R. Hodges, How common are behavioural changes in amyotrophic lateral sclerosis? Amyotrophic Lateral Sclerosis, 2011. 12(1): p. 45-51.

68. Murphy, J.M., R.G. Henry, S. Langmore, et al., Continuum of frontal lobe impairment in amyotrophic lateral sclerosis. Archives of Neurology, 2007. 64(4): p. 530-4.

69. Cerami, C., A. Dodich, N. Canessa, et al., Emotional empathy in amyotrophic lateral sclerosis: a behavioural and voxel-based morphometry study. Amyotrophic Lateral Sclerosis and Frontotemporal Degeneration 2014. 15(1-2): p. 21-29.
70. Meier, S.L., A.J. Charleston, and L.J. Tippett, Cognitive and behavioural deficits associated with the orbitomedial prefrontal cortex in amyotrophic lateral sclerosis. Brain, 2010. 133(11): p. 3444-57.

71. Oh, S.-i., A. Park, H.-J. Kim, et al., Spectrum of Cognitive Impairment in Korean ALS Patients without Known Genetic Mutations. . PLoS ONE [Electronic Resource], 2014. 9(2): p. e87163.

72. Andres, P.L., L.J. Finison, T. Conlon, L.M. Thibodeau, and T.L. Munsat, Use of composite scores (megascores) to measure deficit in amyotrophic lateral sclerosis. Neurology, 1988. 38(3): p. 405-8.

73. McGuire, D., L. Garrison, C. Armon, et al., Relationship of the Tufts Quantitative Neuromuscular Exam (TQNE) and the Sickness Impact Profile (SIP) in measuring progression of ALS. Neurology, 1996. 46(5): p. 1442-4.

74. Goonetilleke, A., H. Modarres-Sadeghi, and R.J. Guiloff, Accuracy, reproducibility, and variability of handheld dynamometry in motor neuron disease. Journal of Neurology, Neurosurgery \& Psychiatry, 1994. 57(3): p. 326-32.

75. Cheah, B.C., S. Vucic, A.V. Krishnan, R.A. Boland, and M.C. Kiernan, Neurophysiological index as a biomarker for ALS progression: validity of mixed effects models. Amyotrophic Lateral Sclerosis, 2011. 12(1): p. 33-8.

76. Felice, K.J., A longitudinal study comparing thenar motor unit number estimates to other quantitative tests in patients with amyotrophic lateral sclerosis. Muscle \& Nerve, 1997. 20(2): p. 179-85.

77. Shefner, J.M., M.L. Watson, L. Simionescu, et al., Multipoint incremental motor unit number estimation as an outcome measure in ALS. Neurology, 2011. 77(3): p. 235-41.

78. Kadrie, H.A., S.K. Yates, H.S. Milner-Brown, and W.F. Brown, Multiple point electrical stimulation of ulnar and median nerves. Journal of Neurology, Neurosurgery \& Psychiatry, 1976. 39(10): p. 973-85.

79. Winhammar, J.M.C., D.B. Rowe, R.D. Henderson, and M.C. Kiernan, Assessment of disease progression in motor neuron disease. Lancet Neurology, 2005. 4(4): p. 229-38.

80. Cedarbaum, J.M., N. Stambler, E. Malta, et al., The ALSFRS-R: a revised ALS functional rating scale that incorporates assessments of respiratory function. BDNF ALS Study Group (Phase III). Journal of the Neurological Sciences, 1999. 169(1-2): p. 13-21.

81. Franchignoni, F., G. Mora, A. Giordano, P. Volanti, and A. Chio, Evidence of multidimensionality in the ALSFRS-R Scale: a critical appraisal on its measurement properties using Rasch analysis. Journal of Neurology, Neurosurgery \& Psychiatry, 2013. 84(12): p. $1340-5$.

82. Gordon, P.H. and Y.K. Cheung, Progression rate of ALSFRS-R at time of diagnosis predicts survival time in ALS. Neurology, 2006. 67(7): p. 1314-5; author reply 1314-5.

83. Kimura, F., C. Fujimura, S. Ishida, et al., Progression rate of ALSFRS-R at time of diagnosis predicts survival time in ALS. Neurology, 2006. 66(2): p. 265-7.

84. Arts, I.M.P., S. Overeem, S. Pillen, et al., Muscle ultrasonography: a diagnostic tool for amyotrophic lateral sclerosis. Clinical Neurophysiology, 2012. 123(8): p. 1662-7.

85. Rutkove, S.B., H. Zhang, D.A. Schoenfeld, et al., Electrical impedance myography to assess outcome in amyotrophic lateral sclerosis clinical trials. Clinical Neurophysiology, 2007. 118(11): p. 2413-8.

86. Turner, M.R., J. Grosskreutz, J. Kassubek, et al., Towards a neuroimaging biomarker for amyotrophic lateral sclerosis. Lancet Neurology, 2011. 10(5): p. 400-3.

87. Puentes, F., J. Topping, J. Kuhle, et al., Immune reactivity to neurofilament proteins in the clinical staging of amyotrophic lateral sclerosis. Journal of Neurology, Neurosurgery \& Psychiatry, 2014. 85(3): p. 274-8.

88. Abel, O., J.F. Powell, P.M. Andersen, and A. Al-Chalabi, ALSoD: A user-friendly online bioinformatics tool for amyotrophic lateral sclerosis genetics. Human Mutation, 2012. 33(9): p. 1345-51. 
89. Renton, A.E., A. Chio, and B.J. Traynor, State of play in amyotrophic lateral sclerosis genetics. Nature Neuroscience, 2014. 17(1): p. $17-23$.

90. Vance, C., B. Rogelj, T. Hortobagyi, et al., Mutations in FUS, an RNA processing protein, cause familial amyotrophic lateral sclerosis type 6. Science, 2009. 323(5918): p. 1208-11.

91. Andersen, P., M. Morita, and R. Brown, Genetics of amyotrophic lateral sclerosis: An overview, in Amyotrophic Lateral Sclerosis, R. Brown, V. Meininger, and M. Swash, Editors. 2000, Martin-Dunitz: London. p. 223-250.

92. Andersen, P.M. and A. Al-Chalabi, Clinical genetics of amyotrophic lateral sclerosis: what do we really know? Nature Reviews Neurology, 2011. 7(11): p. 603-15.

93. Al-Chalabi, A., S. Kwak, M. Mehler, et al., Genetic and epigenetic studies of amyotrophic lateral sclerosis. Amyotrophic Lateral sclerosis \& Frontotemporal Degeneration, 2013. 14 Suppl 1: p. 44-52.

94. Dunckley, T., M.J. Huentelman, D.W. Craig, et al., Whole-genome analysis of sporadic amyotrophic lateral sclerosis. New England Journal of Medicine, 2007. 357(8): p. 775-88.

95. Schymick, J.C., S.W. Scholz, H.-C. Fung, et al., Genome-wide genotyping in amyotrophic lateral sclerosis and neurologically normal controls: first stage analysis and public release of data. Lancet Neurology, 2007. 6(4): p. 322-8.

96. Landers, J.E., J. Melki, V. Meininger, et al., Reduced expression of the Kinesin-Associated Protein 3 (KIFAP3) gene increases survival in sporadic amyotrophic lateral sclerosis. Proceedings of the National Academy of Sciences of the United States of America, 2009. 106(22): p. 9004-9.

97. Consortium, A., K.B. Ahmeti, S. Ajroud-Driss, et al., Age of onset of amyotrophic lateral sclerosis is modulated by a locus on $1 \mathrm{p} 34.1$. Neurobiology of Aging, 2013. 34(1): p. 357.e7-19.

98. Cooper-Knock, J., P.J. Shaw, and J. Kirby, The widening spectrum of C9ORF72-related disease; genotype/phenotype correlations and potential modifiers of clinical phenotype. Acta Neuropathologica, 2014. 127(3): p. 333-45.

99. Al-Chalabi, A. and O. Hardiman, The epidemiology of ALS: a conspiracy of genes, environment and time. Nature Reviews Neurology, 2013. 9(11): p. 617-28.

100. Droppelmann, C., D. Campos-Melo, M. Ishtiaq, K. Volkening, and M. Strong, RNA metabolism in ALS: When normal processes become pathological Amyotrophic Lateral Sclerosis and Frontotemporal Degeneration 2014. 15(5-6): p. 321-336.

101. Strong, M.J., The evidence for altered RNA metabolism in amyotrophic lateral sclerosis (ALS). Journal of the Neurological Sciences, 2010. 288(1-2): p. 1-12

102. Desport, J.C., P.M. Preux, L. Magy, et al., Factors correlated with hypermetabolism in patients with amyotrophic lateral sclerosis. American Journal of Clinical Nutrition., 2001. 74(3): p. 328-34.

103. Ludolph, A.C., 135th ENMC International Workshop: nutrition in amyotrophic lateral sclerosis 18-20 of March 2005, Naarden, The Netherlands. Neuromuscular Disorders., 2006. 16(8): p. 530-8.

104. Desport, J.C., F. Torny, M. Lacoste, P.M. Preux, and P. Couratier, Hypermetabolism in ALS: correlations with clinical and paraclinical parameters. Neurodegenerative Diseases., 2005. 2(3-4): p. 202-7.

105. Sherman, M., A. Pillai, A. Jackson, and T. Heiman-Patterson, Standard equations are not accurate in assessing resting energy expenditure in patients with amyotrophic lateral sclerosis. J. Parenteral Enteral Nutrition, 2004. 28(6): p. 442-446.

106. Armon, C., M.C. Graves, D. Moses, et al., Linear estimates of disease progression predict survival in patients with amyotrophic lateral sclerosis. Muscle \& Nerve, 2000. 23(6): p. 874-82.

107. Cameron, A. and J. Rosenfeld, Nutritional issues and supplements in amyotrophic lateral sclerosis and other neurodegenerative disorders. 2002: p. 631-43.

108. Camu, W., J. Z, C. J, C. C, and B. F. Early Percutaneous Endoscopic Gastrostomy in ALS is a Major FActor Survival for Improving
Survival. in 53rd Annual Meeting of the American Academy of Neurology. 2001. Philadelphia, PA.

109. Desport, J.C., P.M. Preux, C.T. Truong, et al., Nutritional assessment and survival in ALS patients. Amyotrophic Lateral Sclerosis \& Other Motor Neuron Disorders, 2000. 1(2): p. 91-6.

110. Desport, J.C., P.M. Preux, T.C. Truong, et al., Nutritional Status is a prognostic for survival in ALS patients. Neurology, 1999. 53(5): $\mathrm{p}$. 1059-1063.

111. Himes, D., Protein-calorie malnutrition and involuntary weight loss: the role of aggressive nutritional intervention in wound healing. Ostomy/Wound Healing, 1999. 45(3): p. 46-55.

112. Aridegbe, T., R. Kandler, S.J. Walters, et al., The natural history of motor neuron disease: assessing the impact of specialist care. Amyotrophic Lateral sclerosis \& Frontotemporal Degeneration, 2013. 14(1): p. 13-9.

113. Miller, R.G., C.E. Jackson, E.J. Kasarskis, et al., Practice parameter update: the care of the patient with amyotrophic lateral sclerosis: multidisciplinary care, symptom management, and cognitive/behavioral impairment (an evidence-based review): report of the Quality Standards Subcommittee of the American Academy of Neurology. Neurology, 2009. 73(15): p. 1227-33.

114. Rodriguez de Rivera, F.J., C. Oreja Guevara, I. Sanz Gallego, et al., Outcome of patients with amyotrophic lateral sclerosis attending in a multidisciplinary care unit. Neurologia, 2011. 26(8): p. 455-60.

115. Mazzini, L., T. Corra, M. Zaccala, et al., Percutaneous endoscopic gastrostomy and enteral nutrition in amyotrophic lateral sclerosis. Journal of Neurology, 1995. 242(10): p. 695-8.

116. Miller, R.G., C.E. Jackson, E.J. Kasarskis, et al., Practice parameter update: the care of the patient with amyotrophic lateral sclerosis: drug, nutritional, and respiratory therapies (an evidence-based review): report of the Quality Standards Subcommittee of the American Academy of Neurology. Neurology, 2009. 73(15): p. 1218-26.

117. Andersen, P.M., G.D. Borasio, R. Dengler, et al., Good practice in the management of amyotrophic lateral sclerosis: clinical guidelines. An evidence-based review with good practice points. EALSC Working Group. 2007: p. 195-213.

118. Trail, M., N.D. Nelson, J.N. Van, S.H. Appel, and E.C. Lai, A study comparing patients with amyotrophic lateral sclerosis and their caregivers on measures of quality of life, depression, and their attitudes toward treatment options. Journal of the Neurological Sciences., 2003. 209(1-2): p. 79-85.

119. Chio, A., G. Mora, M. Leone, et al., Early symptom progression rate is related to ALS outcome: a prospective population-based study.[see comment]. 2002: p. 99-103.

120. Shaw, A.S., M.A. Ampong, A. Rio, et al., Survival of patients with ALS following institution of enteral feeding is related to pre-procedure oximetry: a retrospective review of 98 patients in a single centre. 2006: p. 16-21.

121. Strong, M.J., A. Rowe, and R.N. Rankin, Percutaneous gastrojejunostomy in amyotrophic lateral sclerosis. Journal of the Neurological Sciences, 1999: p. 128-132.

122. Heffernan, C., C. Jenkinson, T. Holmes, et al., Nutritional management in MND/ALS patients: an evidence based review. Amyotrophic Lateral Sclerosis \& Other Motor Neuron Disorders., 2004. 5(2): p. 72-83.

123. Miller, R.G., C.E. Jackson, E.J. Kasarskis, et al., Practice Parameter update: The care of the patient with amyotrophic lateral sclerosis: Drug, nutritional, and respiratory therapies (an evidence-based review): Report of the Quality Standards Subcommittee of the American Academy of Neurology. Neurology, 2009. 73: p. 12181226.

124. Chio, A., R. Galletti, C. Finocchiaro, et al., Percutaneous radiological gastrostomy: a safe and effective method of nutritional tube placement in advanced ALS. Journal of Neurology, Neurosurgery \& Psychiatry, 2004. 75(4): p. 645-7. 
125. Pacicco, T.J., S. Lindblom, and J. Rosenfeld. Enhancing PEG Tube Placement: A New Device to Maintain Respiratory Function During Endoscopy in ALS Patients. in International Motor Neuron Disease Symposium. 2005. Dubiln, Ireland.

126. Kleopa, K.A., M. Sherman, B. Neal, G.J. Romano, and T. HeimanPatterson, Bipap improves survival and rate of pulmonary function decline in patients with ALS [see comments]. Journal of the Neurological Sciences, 1999. 164(1): p. 82-8.

127. Pinto, A.C., T. Evangelista, M. Carvalho, M.A. Alves, and M.L. Sales Luis, Respiratory assistance with a non-invasive ventilator (Bipap) in MND/ALS patients: survival rates in a controlled trial. Journal of the Neurological Sciences, 1995. 129(Suppl): p. 19-26.

128. Hanayama, K., Y. Ishikawa, and J.R. Bach, Amyotrophic lateral sclerosis. Successful treatment of mucous plugging by mechanical insufflation-exsufflation. American Journal of Physical Medicine \& Rehabilitation, 1997. 76(4): p. 338-9.

129. Senent, C., J.-L. Golmard, F. Salachas, et al., A comparison of assisted cough techniques in stable patients with severe respiratory insufficiency due to amyotrophic lateral sclerosis. Amyotrophic Lateral Sclerosis, 2011. 12(1): p. 26-32.
130. Arens, R., D. Gozal, K. Omlin, et al., Comparison of high frequency chest compression and conventional chest physiotherapy I hospitalized patients with cystic fibrosis. Am J Respir Crit Care Med, 1994. 150: p. 1154-1157.

131. Warwick, W. and L. Hansen, The long term effect of high frequency chest compression therapy on pulmonary complications of cystic fibrosis. Pediatr Pumonol, 1991. 11: p. 265-271.

132. Anagnostou, E., M. Rentzos, T. Alexakis, et al., Volume matters: the influence of different botulinum toxin-A dilutions for sialorrhea in amyotrophic lateral sclerosis.[Erratum appears in Muscle Nerve. 2013 Jul;48(1):155 Note: Evangelos, Anagnostou [corrected to Anagnostou, Evangelos]; Michael, Rentzos [corrected to Rentzos, Michael]; Theodoros, Alexakis [corrected to Alexakis, Theodoros]; Vasiliki, Zouvelou [corrected to Zouvelou, Vasiliki]; Thomas, Zambelis [corrected to Zambelis, Thomas]; Ioannis, Evdokimidis [corrected to Evdokimidis, Ioannis]]. Muscle \& Nerve, 2013. 47(2): p. $276-8$.

133. Jackson, C.E., G. Gronseth, J. Rosenfeld, et al., Randomized double-blind study of botulinum toxin type B for sialorrhea in ALS patients. Muscle \& Nerve, 2009. 39(2): p. 137-43. 